\title{
DETERMINAÇÃO DE Cu, Pb E Zn NO SEDIMENTO DA REGIÃO DO PONTAL DA BARRA, LARANJAL (LAGUNA DOS PATOS, PELOTAS - RS, BRASIL)
}

\author{
Caldas, J. S. ${ }^{1,2} \&$ Sanches Filho, P. J. ${ }^{2 *}$ \\ 1 - Universidade Federal do Rio Grande, FURG, Brasil \\ 2 - Instituto Federal Sul-Rio-Grandense, IFSUL, Brasil \\ *Corresponding author: sanches@pelotas.ifsul.edu.br
}

\begin{abstract}
Caldas, J. S. \& Sanches Filho, P. J. (2013) Determination of $\mathrm{Cu}, \mathrm{Pb}$ e $\mathrm{Zn}$ in sediment of the region of Pontal da Barra, Laranjal (Patos Lagoon, Pelotas - RS, Brasil). Braz. J. Aquat. Sci. Technol. 17(1): 13-18. elSSN $1983-9057$. The region of Pontal da Barra is cited by the Tropical Database (BDT) as a priority area for preservation. In this context, we developed a study aimed at determining the levels of heavy metals such as $\mathrm{Cu}, \mathrm{Pb}, \mathrm{Zn}$ in the sediment of this region, the estuary of Patos Lagoon, located in the São Gonçalo Canal that connects with the Lagoon. The sampling took place in May 2010 , on seven points. Samples were extracted by acid $(3: 1 \mathrm{HCl}: \mathrm{HNO} 3$ and $1 \mathrm{~mL}$ of HClO4$)$ at $90^{\circ} \mathrm{C}$ and the extracts analyzed by flame atomic absorption spectrophotometry. The heavy metal concentrations varied among points: $\mathrm{Cu}-4.4$ to $13.2 \mathrm{mg} \mathrm{kg}^{-1} ; \mathrm{Pb}-11.67$ to $23.21 \mathrm{mg} \mathrm{kg}^{-1} ; \mathrm{Zn}$ of 20.3 to $84.0 \mathrm{mg} \mathrm{kg}^{-1}$. The results show low levels of contamination in comparison with the Canadian Quality Guide for Estuarine Sediments.
\end{abstract}

Keywords: preservation, estuary, heavy metals, contamination, sediments.

\section{INTRODUÇÃO}

A região do Pontal da Barra, na Praia do Laranjal, no Município de Pelotas, situa-se entre a margem direita da Laguna dos Patos e a margem esquerda do Canal São Gonçalo. É uma região referida pela Base de Dados Tropicais (BTD), como área prioritária para a preservação (Fundação André Tosello, 1996), definida como Reserva Particular do Patrimônio Natural (RPPN) pela portaria $n^{\circ} 080 / 99$, do Ministério do Meio Ambiente, possuindo 65,33 ha de área a ser preservada (Brasil, 1999).

Sendo uma área de banhado, constitui um local de berçário para diversas espécies, servindo de peça chave na conservação da biodiversidade (Fundação André Tosello, 1996) Também, serve de controle hidrológico para as regiões vizinhas e atende a comunidade pesqueira local, caracterizando-se como um ambiente de importância econômica, pois é fonte de subsistência para diversas famílias. Possui grande importância paisagística e turística, ainda pouco explorada, onde a sanidade ambiental é imprescindível para a manutenção do ambiente e para o estabelecimento de políticas públicas.

Sendo um local de ligação entre o Canal e a Laguna, suas águas sofrem influência de ambos (Sá, 2006). Quanto à influência do Canal São Gonçalo, sabe-se que este, recebe águas de diferentes arroios e canais que permeiam a zona urbana e zonas rurais de Pelotas, destacando-se o canal Santa Bárbara, o Canal do Pepino e o Arroio Pelotas, os quais recebem resíduos sólidos, esgotos domésticos e águas residu- árias do meio urbano e rural, que são conduzidos à Laguna (Sá, 2006).

Já a Laguna, causa influência, por esta região fazer parte da sua porção estuarina. É um ambiente caracterizado por flutuações na salinidade, o que influencia diretamente a distribuição de contaminantes entre a água, biota e sedimento (Quináglia, 2006). Além disso, a ação dos ventos e o desnível das águas podem influenciar o sentido da corrente de fluxo (Stumm \& Morgan, 1996; Sá, 2006).

Através de todas essas possíveis influências, muitos poluentes ambientais como os metais pesados podem contaminar este ambiente trazendo sérias consequências para a vida deste ecossistema e provocar efeitos adversos nos seres humanos (Sá, 2006).

A concentração de elementos-traço em águas superficiais é consequência de alguns processos como: a geoquímica das rochas e solos de origem da bacia; a poluição antropogênica a partir de resíduos ou deposição atmosférica; as reações químicas, através da adsorção em partículas e outras superfícies, e a deposição nos sedimentos (Cesar et al., 2007).

Com isso, a análise dos sedimentos torna-se um aspecto importante a ser considerado. Uma vez que, o sedimento tem capacidade de acumular elementos-traços, atuar como agente transportador e ainda, ser possível fonte de contaminação (Cotta et al., 2006). A determinação de metais em sedimentos permite detectar o grau de contaminação a que a água e todo o ecossistema, estão sujeitos (Robaina et al.,2002). Tornando-se possível a adequação de medidas de prevenção evitando-se a futura poluição do ambiente. 


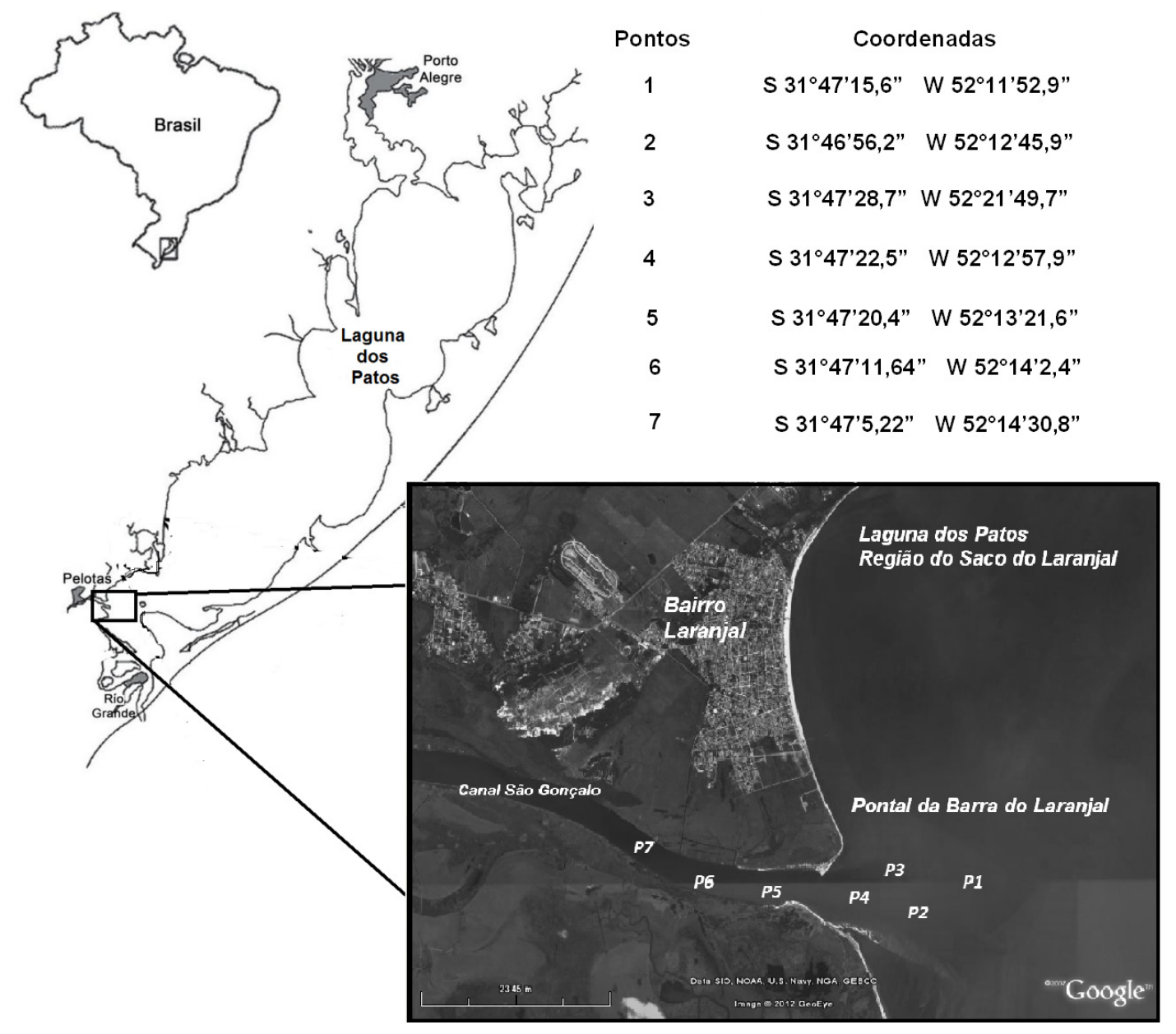

Figura 1 - Localização da Área de Estudo. Fonte: Google Earth, Novembro de 2010, modificado pelo autor.

Desta forma, este trabalho teve por objetivo determinar dos níveis de metais pesados $(\mathrm{Cu}, \mathrm{Pb}$, $\mathrm{Zn)}$ na região do Pontal da Barra, visando a caracterização das condições ambientais frente a estes contaminantes.

\section{MATERIAL E MÉTODOS}

A pesquisa foi realizada na localidade do Pontal da Barra, no bairro Laranjal, distante aproximadamente $15 \mathrm{~km}$ do centro da cidade de Pelotas - 31 ${ }^{\circ} 46^{\prime} 19^{\prime \prime} \mathrm{S} \mathrm{e}$ $52^{\circ} 20^{\prime} 33^{\prime \prime} \mathrm{O}$. O local é delimitado a Leste pela Laguna dos Patos, ao Sul é tangenciado pelo Canal São Gonçalo e ao Norte encontra-se a área urbanizada do bairro Laranjal. A pesquisa experimental foi realizada no Laboratório de Análise de Contaminantes Ambientais (LACA) do Instituto Federal de Educação, Ciência e Tecnologia sul-rio-grandense (IFSul).

A amostragem foi desenvolvida no início do mês de maio de 2010 e percorreu 7 pontos, da saída do saco do laranjal até a entrada no Canal São Gonçalo, dentre estes, os pontos 1, 2, 3 e 4 foram amostrados no final do Saco do Laranjal e os pontos 5, 6 e 7 foram amostrados na porção final do Canal São Gonçalo, as coordenadas geográficas e as localização dos pontos encontra-se na figura 1.

O sedimento coletado foi utilizado na determinação de metais, matéria orgânica, umidade e granulometria. Durante a amostragem foram realizadas in loco análises na água do $\mathrm{pH}$, condutividade e temperatura.

As amostras de sedimento $(0-5 \mathrm{~cm})$ foram coletadas como uma draga de aço inoxidável do tipo "Van Veen", assim como, indica Mozeto (2007). O material para análise foi coletado do interior do sedimento, na porção central, evitando-se a contaminado pelo metal da draga. Este, por sua vez, foi armazenado em potes de polietileno previamente descontaminados e, posteriormente, acondicionados em ambiente refrigerado à aproximadamente $4^{\circ} \mathrm{C}$.

As amostras para determinação dos metais foram secas em estufa a $60^{\circ} \mathrm{C}$ por 48 horas, seguida por maceração e peneiramento, sendo a fração $<63 \mu \mathrm{m}$ escolhida para o tratamento químico de extração.

Os metais foram extraídos através de digestão ácida pseudototal (para determinação dos metais mais 
biodisponíveis no sedimento). A $2 \mathrm{~g}$ da fração menor que $63 \mu \mathrm{m}$ do sedimento, foram adicionados $4 \mathrm{~mL}$ de água régia $\left(3: 1 \mathrm{HCl}^{-\mathrm{HNO}_{3}}\right), 1 \mathrm{~mL}$ de $\mathrm{HClO}_{4}$ e $4 \mathrm{~mL}$ de água Mili-Q, com aquecimento por 30 minutos a $90^{\circ} \mathrm{C}$, sendo as extrações realizadas no mínimo em triplicata (Hortellani, et al., 2008). A solução resultante foi filtrada e transferida para um balão volumétrico de $50 \mathrm{~mL}$, completando-se o seu volume com água ultra pura. A vidraria utilizada no tratamento e armazenamento das amostras foi descontaminada em uma solução de $\mathrm{HNO}_{3}$ a $10 \%(\mathrm{v} / \mathrm{v})$, por $24 \mathrm{hs}$, seguida por secagem em estufa a $105^{\circ} \mathrm{C}$.

A curva de calibração foi obtida a partir da diluição soluções padrão marca Tritisol de $1000 \mathrm{mg} / \mathrm{L}$ dos metais analisados, nas concentrações de 0,2 a 2,0 $\mathrm{mg} \mathrm{L}^{-1}$ para o zinco, chumbo e cobre, sendo obtida a equação para cada metal. Após a extração, os analitos foram determinados por Espectrofotometria de Absorção Atômica em chama em um espectrofotômetro da marca GBC 932 Plus, utilizando acetileno como gás inerte, com valores de comprimento de onda de cada elemento e corrente da lâmpada de, respectivamente, $\mathrm{Pb}: 217,0 \mathrm{~nm}$ e $5,0 \mathrm{~mA}$; Cu: 324,7 $\mathrm{nm}$ e 3,0mA; Zn: 213,9 nm e 5,0 mA. As leituras das amostras foram feitas em triplicata.

Para a certificação analítica foi utilizada uma amostra de referência NMCR\#4 para solo e sedimento, obtido da Ultra Scientifc Analytical Solutions. Os limites de detecção (LD) e quantificação (LQ) foram encontrados a partir de dez medidas do branco da amostra no espectrofotômetro. Calculando-se o desvio padrão e multiplicando-se por 3 para o LD e por 10 para o LQ (IUPAC, 1997; Castro et al., 2008).

Dentre os ensaios que foram executados em laboratório; o ensaio de teor de umidade foi realizado pelo método gravimétrico, a partir de $20 \mathrm{~g}$ da amostra e secagem em estufa a $105^{\circ} \mathrm{C}$ durante $12 \mathrm{hs}$ com posterior pesagem. Este procedimento foi realizado segundo uma modificação do método descrito pela EMBRAPA (1997) em seu manual, a modificação consistiu em realizar o procedimento em $12 \mathrm{hs}$, não em $24 \mathrm{hs}$ como descrito pelo método.
O teor de matéria orgânica foi determinado através do método de perda de peso (Walkley \& Black, 1934 apud Angonesi, 2005) por calcinação em mufla à $550^{\circ} \mathrm{C}$ por $4 \mathrm{hs}$ e a análise granulométrica foi feita pelo método padrão peneira, classificando o tamanho dos grãos de acordo com a escala Wentworth, método descrito por Surguio (1973).

\section{RESULTADOS E DISCUSSÃO}

A tabela 1 apresenta os dados físico-químicos para água e caracteriza o sedimento da região amostrada, a cerca do teor de umidade e de matéria orgânica. Observa-se que, a umidade variou de 58,8 a $71,1 \%$ e o teor de umidade dos pontos 1 a 4 foi maior que nos demais pontos, da mesma forma, o teor de matéria orgânica foi maior nos pontos de 1 a 5 . Isso indica uma redução na turbulência, favorecendo a deposição de material particulado e consequentemente, a possibilidade de encontrarmos maiores níveis de contaminação, uma vez que, os contaminantes se adsorvem as partículas orgânicas do sedimento, e estas, por sua vez, depositam-se em regiões de sedimentos finos (Noale, 2007; Torres, 2009).

Em relação à umidade, a retenção da água no solo ocorre devido a ação de forças de adesão das moléculas de água pelas partículas sólidas e coesão entre as próprias moléculas de água (Marenco \& Lopes, 2005). Quanto menor é a granulometria do sedimento, maior é a área superficial onde a água é adsorvida, consequentemente, maior teor de umidade (Marenco \& Lopes, 2005. p.169), tal comportamento é refletido nos resultados obtidos.

A tabela 2 apresenta a diferença granulométrica entre os pontos em estudo, observa-se que os pontos de maneira geral apresentam uma granulometria predominantemente fina, aumentando a probabilidade de acumulação de contaminantes, devido à elevada área superfícial específica (Cotta, 2006). Dentro do canal a granulometria aumenta gradativamente, assim como é menor a matéria orgânica e a umidade do sedimento.

Tabela 1 - Teor de umidade e teor de matéria orgânica para o sedimento e parâmetros físico-químicos da água nos pontos amostrados.

\begin{tabular}{cccccc}
\hline \hline & \multicolumn{2}{c}{ Sedimento } & \multicolumn{3}{c}{ Água } \\
Pontos & $\begin{array}{c}\text { Umidade } \\
(\%)\end{array}$ & $\begin{array}{c}\text { MO } \\
(\%)\end{array}$ & $\begin{array}{c}\text { Condutividade } \\
\left(\mu \text { S/cm à 25 }{ }^{\circ} \mathrm{C}\right)\end{array}$ & $\mathrm{pH}$ & $\begin{array}{c}\text { Temperatura } \\
\left({ }^{\circ} \mathrm{C}\right)\end{array}$ \\
\hline 1 & 68,8 & 9,4 & 298,0 & 7,5 & 18,0 \\
2 & 68,2 & 9,9 & 301,1 & 7,5 & 18,0 \\
3 & 71,1 & 11,5 & 256,8 & 7,5 & 18,1 \\
4 & 68,0 & 11,9 & 168,8 & 8,0 & 18,0 \\
5 & 60,6 & 10,8 & 131,8 & 8,2 & 18,1 \\
6 & 63,8 & 5,3 & 132,8 & 8,1 & 18,1 \\
7 & 58,0 & 4,5 & 159,8 & 8,1 & 18,1 \\
\hline \hline
\end{tabular}


Tabela 2 - Granulometria do sedimento nos pontos, em amostras de $20 \mathrm{~g}$ de sedimento. Valores em percentual de massa.

\begin{tabular}{cccccccc}
\hline \hline Pontos & $\begin{array}{c}\text { Grão } \\
\mathbf{2 m m}\end{array}$ & $\begin{array}{c}\text { Areia } \\
\text { Muito } \\
\text { Grossa } \\
\mathbf{1 m m}\end{array}$ & $\begin{array}{c}\text { Areia } \\
\text { Grossa } \\
\mathbf{7 1 0} \boldsymbol{\mu m}\end{array}$ & $\begin{array}{c}\text { Areia } \\
\text { Média } \\
\mathbf{5 0 0} \boldsymbol{\mu m}\end{array}$ & $\begin{array}{c}\text { Areia } \\
\text { Fina } \\
\mathbf{2 5 0} \boldsymbol{\mu m}\end{array}$ & $\begin{array}{c}\text { Areia } \\
\text { Muito } \\
\text { Fina } \\
\mathbf{1 2 5 \mu m}\end{array}$ & $\begin{array}{c}\text { Fundo } \\
<\mathbf{1 2 5} \boldsymbol{\mu m}\end{array}$ \\
\hline 1 & 0,0 & 8,2 & 20,6 & 14,5 & 22,9 & 15,4 & 18,0 \\
2 & 0,7 & 3,3 & 19,0 & 11,2 & 25,2 & 17,9 & 22,3 \\
3 & 0,5 & 1,5 & 11,7 & 13,6 & 19,8 & 31,2 & 22,2 \\
4 & 0,0 & 6,7 & 10,8 & 10,3 & 21,0 & 22,4 & 28,9 \\
5 & 0,0 & 5,4 & 6,9 & 19,4 & 16,9 & 17,7 & 33,6 \\
6 & 0,8 & 2,7 & 7,7 & 10,3 & 29,4 & 20,5 & 28,7 \\
7 & 0,2 & 3,5 & 9,8 & 11,3 & 28,8 & 20,3 & 25,7 \\
\hline \hline
\end{tabular}

Quanto aos parâmetros físico-químicos avaliados, a temperatura foi praticamente constante em todos os pontos, em torno de $18^{\circ} \mathrm{C}$ e observou-se que as águas na região do Pontal da Barra, apresentam-se de acordo com os parâmetros estabelecidos pela resolução CONAMA $n^{\circ} 357 / 2005$ para águas salobras, classe 2 .

A tabela 3 apresenta os resultados da determinação dos metais pesados no sedimento, observa-se que os analitos cobre, chumbo e zinco, foram encontrados em todos os pontos amostrados. Dentre os analitos estudados, o zinco foi majoritário nas amostras. Comparando-se os resultados com os valores definidos pelo Guia de Qualidade de sedimentos para proteção da vida aquática do Canadá (CCME EPC-98E, 1999), observa-se que os valores obtidos, estão abaixo do TEL (threshold effect level), indicando baixa contaminação, ou seja, raramente ocorrerão efeitos biológicos danosos em função destes xenobióticos.

A tabela 4 apresenta os níveis de metais no sedimento de referência, os valores encontrados e os resultados da matriz de referência, os resultados demonstram que o método de extração foi adequado ao experimento os valores de recuperação variaram de $75,02 \%$ a $103,49 \%$ sendo considerados por (U. S. EPA, 1996) como satisfatórios não sendo necessário nenhum tipo de correção nas amostras.

Dentre os pontos amostrados, os pontos 1 a 5 , que e representam respectivamente, o final e a chegada do Canal São Gonçalo na Laguna, apresentam maior contaminação, dado que reforça a estreita relação destes metais à matéria orgânica presente em maior quantidade junto a sedimentos finos.

Curiosamente, o ponto 5 apresentou maiores níveis de chumbo e zinco, tal comportamento pode ser justificado através a um canal perpendicular ao São Gonçalo que drena o bairro do Laranjal, desaguando neste ponto, sendo uma possível fonte de contaminação.

Os valores encontrados nas análises estão de acordo com os backgrounds para o estuário da Laguna dos Patos (Niencheski et al., 2002) e com os backgrounds para sedimentos mundiais (Bowen, 1979).

Tabela 3 - Níveis de $\mathrm{Cu}, \mathrm{Pb}, \mathrm{Zn}$ nos sedimentos da região do Pontal da Barra, seus respectivos desvios padrões relativos(\%), limites de detecção(LD) e quantificação (LQ), coeficientes angular (a) e linear (b) das suas respectivas curvas de calibração e padrões.

\begin{tabular}{|c|c|c|c|}
\hline Pontos & \multicolumn{3}{|c|}{$\left(\mathrm{mg} \mathrm{Kg}^{-1}\right) \pm \mathrm{RSD}(\%)$} \\
\hline 1 & $12,2 \pm 2,1$ & $12,7 \pm 2,3$ & $35,7 \pm 2,8$ \\
\hline 2 & $13,1 \pm 3,5$ & $13,9 \pm 3,6$ & $36,4 \pm 4,1$ \\
\hline 3 & $13,2 \pm 1,0$ & $14,6 \pm 3,2$ & $37,7 \pm 1,1$ \\
\hline 4 & $8,5 \pm 4,8$ & $17,52 \pm 4,9$ & $29,2 \pm 2,2$ \\
\hline 5 & $5,2 \pm 6,4$ & $23,21 \pm 6,4$ & $84,0 \pm 3,9$ \\
\hline 6 & $4,4 \pm 5,7$ & $11,67 \pm 2,1$ & $20,3 \pm 7,3$ \\
\hline 7 & $5,1 \pm 4,6$ & $12,8 \pm 6,8$ & $22,2 \pm 3,8$ \\
\hline a & 0,052 & 0,0269 & 0,161 \\
\hline b & 0,004 & 0,0094 & 0,003 \\
\hline $\mathbf{R}^{2}$ & 0,998 & 0,994 & 0,998 \\
\hline $\mathbf{L Q}\left(\mathrm{mg} \mathrm{Kg}^{-1}\right)$ & 4,40 & 3,93 & 3,93 \\
\hline LD $\left(\mathrm{mg} \mathrm{Kg}^{-1}\right)$ & 0,40 & 0,35 & 0,36 \\
\hline \multicolumn{4}{|c|}{ Padrões $\left(\mathrm{mg} \mathrm{Kg}^{-1}\right)$} \\
\hline TEL $^{(1)}$ & 18,7 & 30,2 & 124,0 \\
\hline TEL $^{(2)}$ & 35,7 & 35,0 & 123,0 \\
\hline PEL $^{(3)}$ & 108,0 & 112,0 & 271,0 \\
\hline $\mathrm{PEL}^{(4)}$ & 197,0 & 91,3 & 315,0 \\
\hline BGLP $^{(5)}$ & 20,0 & 13,4 & 70,4 \\
\hline BGM $^{(6)}$ & 33,0 & 19,0 & 95,0 \\
\hline
\end{tabular}

1-4 Níveis padrões do Guia de Qualidade dos Sedimentos Estuarinos do Canadá (CCME EPC-98E, 1999)

(1) Threshold Effect Level. Valor abaixo do qual raramente ocorre efeitos biológicos (Água salobra).

(2) Threshold Effect Level. Valor abaixo do qual raramente ocorre efeitos biológicos (Água doce).

(3) Probable Effect Level. Valor acima do qual efeito adverso é esperado (Água salobra).

(4) Probable Effect Level. Valor acima do qual efeito adverso é esperado (Água doce).

(5) BGLP - Background estuário da L. Patos (Niencheski et al., 2002)

(6) BGM - Background sedimentos mundiais (Bowen, 1979) 
Tabela 4 - Níveis de metais no sedimento de referência em $\left(\mathrm{mg} \mathrm{Kg}^{-1}\right)$,valores encontrados e corrigidos - resultados certificados da matriz de referência NMCR\#4 em $\left(\mathrm{mg} \mathrm{Kg}^{-1}\right)$. Rec\% - percentual de recuperação.

\begin{tabular}{lccc}
\hline \hline \multicolumn{4}{c}{ Sedimento de Referência } \\
Metais & $\mathbf{C u}$ & $\mathbf{P b}$ & $\mathbf{Z n}$ \\
\hline Valor encontrado & $37,67 \pm 5,87$ & $97,31 \pm 15,16$ & $92,27 \pm 0,12$ \\
Valor correto & 36,4 & 95,3 & 133,5 \\
Rec\% & 103,49 & 102,11 & 75,02 \\
\hline \hline
\end{tabular}

\section{CONCLUSÕES}

Podemos concluir que nesta amostragem desenvolvida no mês de Maio de 2010 , no período de Outono, foram encontrados os analitos $\mathrm{Cu}, \mathrm{Pb}$ e $\mathrm{Zn}$ em concentrações consideradas normais. Dos metais analisados destacam-se o Zn, em maior concentração, seguido do chumbo, metal sem função biológica e de fácil bioconcentração e biomagnificação na cadeia trófica. No entanto, cabe ressaltar que, esta área de estudo merece o desenvolvimento de pesquisas de monitoração sazonais, pois é uma área influenciada por correntes de fluxo, pelos ventos, pela salinidade da Laguna dos Patos e pelo Canal São Gonçalo, podendo sofrer variações destes contaminantes durante o ano. Pode observar-se também que atividades antrópicas podem ser contribuintes para a contaminação por esses elementos na região do Pontal da Barra do Laranjal, influenciando diretamente na qualidade das águas que desembocam laguna, sendo de suma importância um contínuo monitoramento das condições ambientais desta importante área. Os resultados podem contribuir para alertar quanto aos cuidados a serem tomados em áreas de preservação, buscando-se acompanhar alterações futuras e reduzir o impacto ambiental.

\section{REFERÊNCIAS}

Angonesi, L. G. 2005. Dinâmica de curto prazo da macrofauna bentônica em uma enseada da lagoa dos patos: efeitos antrópicos e mecanismos de persistência e resiliência. Tese de Doutorado. Fundação Universidade Federal do Rio Grande - FURG. 163p.

Bowen, H. J. M. 1979. Environmental geochemistry of the elements. Academic Press, London, 333p.

Brasil. Intituto Brasileiro do Meio Ambiente e dos Recursos Naturais Renováveis - IBAMA. Portaria $N^{\circ} 80-N$, de 22 de Setembro de 1999. RPPN Pontal da Barra. Diário Oficial da República Federativa do Brasil, Brasília, NN183-E, 23 de Setembro de 1999, p. 45, Seção I. ICMBio - SIMRPPN. Lista de RPPN criadas.

Castro, I. M.; Anjos, M. R.; Oliveira, E. S. 2008. Determinação do Isotiocianato de Bensila em
Carica papaya utilizando cromatografia gasosa com detectors seletivos. Rio de Janeiro, Brasil. Química Nova 31(8): 1-10pp.

CCME - Canadian Council of Ministers of Environment. 1999. Protocol for the Derivation of Canadian Sediment Quality Guidelines for the Protection of Aquatic Life. CCME EPC-98E. Ottawa, Canadá.

Cesar, A.; Choueri, R.B.; Riba, I.; Morales-Caselles, C.; Pereira, C.D.S.; Santos, A.R. ; Abessa, D.M.S.; DelValls, T.A. 2007. Comparative sediment quality assessment in different littoral ecosystems from Spain (Gulf of Cadiz) and Brazil (Santos and São Vicente estuarine system), Environment International 33: 429-435pp.

CONAMA, 2005. Classificação dos corpos de água e diretrizes ambientais para o seu enquadramento, bem como estabelece as condições e padrões de lançamento de efluentes, e dá outras providências. Resolução №357, de Março de 2005.

Cotta, J. A. O.; Rezende, M. O. O.; Piovani, M. R. 2006. Avaliação do Teor de Metais em Sedimento do Rio Betari no Parque Estadual Turística do alto Ribeira - PETAR, São Paulo, Brasil. Quimica Nova, 29 (1): 40-45.

EMBRAPA, 1997. Manual de Métodos de Análise de Solo. Ed. Europa, Rio de Janeiro. 212p.

Fundação André Tosello. Base de Dados Tropicais (BDT) 1996. Padrões de Biodiversidade da mata Atlântica do Sudeste e Sul do Brasil.

Hortellani, M. A.; Sarkis, J. E. S.; Abessa, D. M. S.; Souza, E. C. M. 2008. Avaliação da contaminação por elementos metálicos dos sedimentos do Estuário Santos - São Vicente. Química Nova. 31(1): 1-10pp.

IUPAC - International Union of Pure and Applied Chemistry, 1997. Chemistry Compendium of Chemical Terminology. 2nd Edition. (The "Gold Book"). Compiled by McNaught A. D. and Wilkinson A. Blackwell Scientific Publications, Oxford.

Marenco, R. A. \& Lopes, N. F. 2005. Fisiologia Vegetal; fotossíntese, respiração, relações hídricas e nutrição mineral. In: Marenco, R. A. \& Lopes, N. F. Relações Hidricas; Nutrição Mineral. UFV, Viçosa, 165-331pp.

Mozeto, A. A. 2007. Sedimentos e Particulados Lacustres:Amostragem eAnálises Biogeoquímicas. 
In: Bicudo, C. E. de M., BICUDO, D. de C. Amostragem em Limnologia. Rima, São Carlo-SP. 295-317pp.

Niencheski, L. F. H.; Baraj, B.; França, R. G.; Mirlean, N. 2002. Lithium as a normalizer for assessment of anthropogenic metal contamination of sediments of southern área of Patos Lagoon. Aquatic Ecosystem Health and Management, 5(4), 473-483pp.

Noale, R. Z. 2007. Avaliação do Risco Ambiental em sedimentos dos Lagos do Riacho Cambé em Londrina pela Distribuição de Metais. Tese de Mestrado. Universidade de Londrina. 84p.

Quináglia, G. A. 2006. Caracterização dos Níveis Basais de Concentração de Metais nos Sedimentos do Sistema Estuarino da Baixada Santista. Tese de Doutorado. Instituto de Química, Universidade de São Paulo-USP. 269p.

Robaina, L. E.; Formoso, M. L. L.; Pires, C. A. da F. 2002. Metais pesados nos sedimentos de corrente, como indicadores de risco ambiental - Vale do Rio dos Sinos, RS, Brazil, Revista do Instituto Geológico, 23: 35-47.
Sá, M. U. 2006. Avaliação da Mutagenicidade das Águas do Canal São Gonçalo, Pelotas, RS, 2005. Monografia de Conclusão de Curso. Instituto de Biologia, Universidade Federal de Pelotas UFPEL. 60p

Stumm, W. Morgan, J. J. Aquatic chemistry: chemical equilibria and rates in natural Waters. 3rd ed. New York, John Wiley \& Sons Inc., 1996. 1022p.

Surguio, K. 1973. Introdução à sedimentologia. Edgard Blücher- EDUSP, São Paulo, 317p.

Torres, R. F. 2009. Disponibilidade dos Metais Cobre e Chumbo em um Canal de Maré Receptor de Efluentes de Carcinicultura. Tese de Mestrado. Universidade federal do Ceará. $134 p$.

US. EPA. 1996. Inductively Caupled Plasma - Atomic Absorption Emission Spectrometry, Method 6010B. Revision 2.
Submetido: Novembro/2011 Revisado: Outubro/2012 Aceito: Fevereiro/2013 